\title{
Application of the coupled cluster method to the Jaynes-Cummings model without the rotating-wave approximation
}

\author{
R. F. Bishop* and N. J. Davidson ${ }^{\dagger}$ \\ Department of Physics, University of Manchester Institute of Science and Technology (UMIST), P.O. Box 88, Manchester M60 1QD, \\ United Kingdom \\ R. M. Quick \\ Department of Physics, University of Pretoria, Pretoria 0002, South Africa \\ D. M. van der Walt \\ Department of Physics, VISTA University (Mamelodi Campus), Private Bag X 1311, Silverton 0127, South Africa
}

(Received 12 August 1996)

\begin{abstract}
We investigate the Rabi Hamiltonian (or the Jaynes-Cummings model without the rotating-wave approximation), describing the coupling of a single mode of electromagnetic radiation to a two-level system, by means of the coupled cluster method. We find strong evidence for a second-order quantum phase transition. [S1050-2947(96)51112-1]
\end{abstract}

PACS number(s): 42.50.Lc, 42.50.Hz, 32.90.+a, 31.15.Dv

Models of a single mode of a quantized electromagnetic field interacting with a two-level system have long been the subject of considerable interest within the quantum optics community $[1,2]$ as well as, more recently, in the field of quantum chaos [3]. However, at a more basic level, such models describe the interaction between bosons and fermions, and, as such, may provide insights into our understanding of many other areas of physics.

One of the simplest models consists of a single bosonic mode interacting with a two-level system in the dipole approximation, where the Hamiltonian is given by

$$
H=\frac{1}{2} \omega_{0} \sigma^{z}+\omega b^{\dagger} b+g\left(\sigma^{+}+\sigma^{-}\right)\left(b^{\dagger}+b\right),
$$

where $\sigma^{z}, \sigma^{+}$, and $\sigma^{-}$are Pauli matrices, and $b$ and $b^{\dagger}$ are bosonic annihilation and creation operators, respectively, obeying the commutation relation $\left[b, b^{\dagger}\right]=1$. There is a conserved parity $\Pi$ associated with the Hamiltonian (1),

$$
\Pi=\exp [i \pi(N)], \quad N \equiv b^{\dagger} b+\frac{1}{2}\left(\sigma^{z}+1\right) .
$$

There is no proof that the Rabi Hamiltonian (1) is integrable, despite strong numerical indications that this may in fact be true [4], although it definitely becomes so if the rotatingwave approximation (RWA) is made, to yield the JaynesCummings model [1]. In this approximation the counterrotating terms $g\left(b^{\dagger} \sigma^{+}+b \sigma^{-}\right)$are neglected, in which case $\left[H_{\mathrm{RWA}}, N\right]=0$ and the model is exactly soluble by a series of diagonalizations of $2 \times 2$ matrices.

Until now, most calculations based on (1) have made use of the RWA for applications where $g / \omega \ll 1$, and of diago-

\footnotetext{
*Electronic address: R.F.Bishop@umist.ac.uk

$\dagger$ Present address: Department of Physics, University of Pretoria, Pretoria 0002, South Africa.
}

nalization in large but finite vector spaces for $g / \omega \sim 1$. In the present work, we apply one of the most powerful methods of quantum many-body theory, namely the coupled cluster method (CCM), to the Hamiltonian of Eq. (1). The CCM has been successfully applied in such diverse fields of physics as nuclear structure, lattice gauge and continuum field theories, spin and electron lattice models, and quantum hydrodynamics, as well as being widely used in quantum chemistry. A recent application that is in much the same spirit as our work here is to the linear $E-e$ Jahn-Teller effect [5]. The CCM has been described in detail elsewhere [6], and we shall only briefly describe the method here.

The CCM ansatz for the ground-state wave function $|\Psi\rangle$ is given by

$$
|\Psi\rangle=\exp [S]|\Phi\rangle, \quad S=\sum_{I} s_{I} C_{I}^{\dagger}
$$

in terms of a (normalized) model state or cyclic vector $|\Phi\rangle$ and a corresponding complete set of commuting multiconfigurational creation operators $\left\{C_{I}^{\dagger}\right\}$ defined with respect to it, such that $C_{I}|\Phi\rangle=0=\langle\Phi| C_{I}^{\dagger}$. The Schrödinger equation $H|\Psi\rangle=E_{g}|\Psi\rangle$ can then be rewritten in terms of the similarity transformed Hamiltonian as $\exp [-S] H \exp [S]|\Phi\rangle=E_{g}|\Phi\rangle$, so that $E_{g}=\langle\Phi|\exp [-S] H \exp [S]| \Phi\rangle$. The bra ground state $\langle\widetilde{\Psi}|$, which in the CCM is not the manifest Hermitian conjugate of $|\Psi\rangle$, can be parametrized in two ways [7]. In the so-called normal CCM (NCCM) used here,

$$
\langle\widetilde{\Psi}|=\langle\Phi| \widetilde{S} \exp [-S], \quad \widetilde{S}=1+\sum_{I} \widetilde{s_{I}} C_{I}
$$

We note that $\langle\widetilde{\Psi} \mid \Psi\rangle=\langle\Phi \mid \Phi\rangle=1$.

If we define $\bar{H}=\langle\widetilde{\Psi}|H| \Psi\rangle$, the NCCM coefficients $\left\{s_{I}, \widetilde{s_{I}}\right\}$ can be found from the variational conditions $\left(\partial \bar{H} / \partial s_{I}\right)=0=\left(\partial \bar{H} / \partial \widetilde{s_{I}}\right)$. Note that the fact that $\langle\widetilde{\Psi}|$ is not the manifest Hermitian conjugate of $|\Psi\rangle$ means that an ap- 
proximate CCM result for $E_{g}$ does not necessarily provide an upper bound for the true ground-state energy.

For our model state for $\omega_{0}>0$ we use the $g=0$ ground state of (1), $|\Phi\rangle=|0\rangle|\downarrow\rangle$, where the first ket refers to the boson ket in the occupation number representation and the second to the two-level system's state (upper or lower state). Note that this state has positive parity. The correlation operator is given by a sum of two terms,

$$
\begin{gathered}
S=S_{1}+S_{2} ; \\
S_{1}=\sum_{n=1}^{\infty} S_{n}^{(1)}\left(b^{\dagger}\right)^{n}, \quad S_{2}=\sum_{n=1}^{\infty} S_{n}^{(2)}\left(b^{\dagger}\right)^{n-1} \sigma^{+} .
\end{gathered}
$$

It is straightforward to show that the ground-state energy is given by $E_{g}=-\omega_{0} / 2+4 g\left(S_{1}^{(1)} S_{1}^{(2)}+S_{2}^{(2)}\right)$. For states of positive parity, terms in $S$ with $n$ odd are zero. It is important to note that the equations for the coefficients $\left\{S_{n}^{(1)}\right\}$ and $\left\{S_{n}^{(2)}\right\}$ are invariant under the substitutions $S_{n}^{(1)} \rightarrow(-1)^{n} S_{n}^{(1)}$ and $S_{n}^{(2)} \rightarrow(-1)^{n} S_{n}^{(2)}$. This implies that positive-parity states are unique, while mixed-parity states are always doubly degenerate.

Although it is simple to show that the nested commutator expansion $\exp [-S] H \exp [S]=H+[H, S]+\frac{1}{2 !}[[H, S], S]+\cdots$ terminates naturally at third order in $S$ for the above choice of model state and correlation operator, we have to truncate the sums in $S$ to obtain a finite set of equations for the coefficients $\left\{S_{n}^{(1)}\right\}$ and $\left\{S_{n}^{(2)}\right\}$. We will denote the scheme in which both $S_{1}$ and $S_{2}$ truncate at $n=N$ as the SUB- $N$ approximation. Near resonance $\left(\omega \approx \omega_{0}\right)$, this corresponds to an energy cutoff of approximately $N \hbar \omega$.

The Hamiltonian (1) has some exact limits. For zero coupling $(g=0)$ and $\omega_{0}>0$, the model state $|\Phi\rangle$ is the exact ground state with $E_{g}(g=0)=-\omega_{0} / 2$. For the case of two degenerate levels $\left(\omega_{0}=0\right)$, the positive- and negative-parity ground states are degenerate, implying that the ground state does not have good parity in this limit for any coupling. The exact ground states are of SUB-1 form, with $S_{1}^{(1)}= \pm 2 \mathrm{~g} / \omega$ and $S_{1}^{(2)}=\mp 1 / 2$ the only nonzero coefficients. The ground states are thus coherent boson states multiplied by eigenfunctions of $\sigma^{x}$ :

$$
|\Psi\rangle=\mathcal{N} \exp \left[ \pm \frac{2 g}{\omega} b^{\dagger}\right]|0\rangle(|\downarrow\rangle \mp|\uparrow\rangle),
$$

where $\mathcal{N}$ is a normalization constant. The ground-state energy in this case is given by $E_{g}\left(\omega_{0}=0\right)=-4 g^{2} / \omega$.

The case $\omega_{0}=0$ is important because the Hamiltonian with a finite value for $\omega_{0}$ goes over to this case as $g \rightarrow \infty$. This implies that the ground state has changed from being unique to being doubly degenerate, and thus hints at the presence of a parity-breaking phase transition. The results of our CCM analysis bear this out.

We turn now to our results for the ground-state energy for the Hamiltonian (1) with $\omega_{0}>0$. Since the ground state at $g=0$ has positive parity, we expect the same to be true for small values of the coupling. For this reason, we will restrict our calculations to SUB- $N$ approximations where $N$ is even. Starting from $g=0$, where the model state is the exact ground state, we can solve for ever increasing values of the

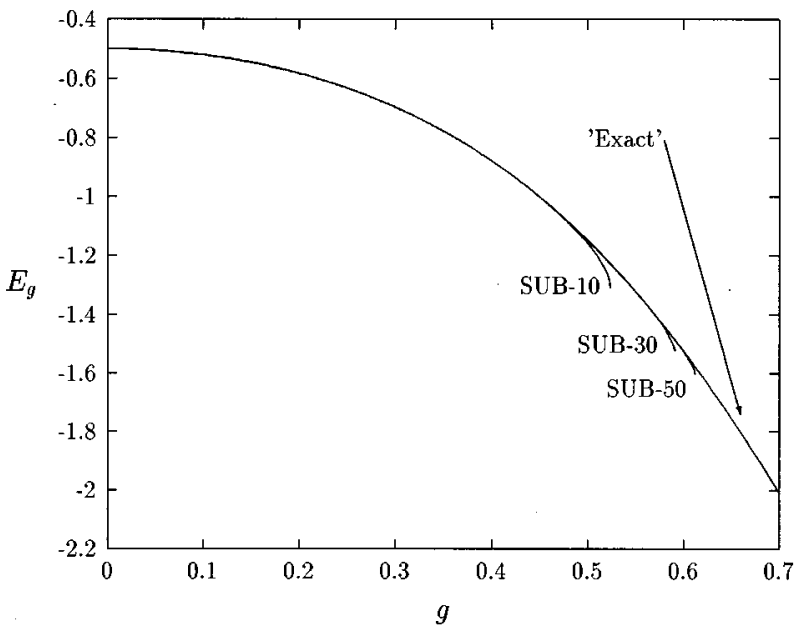

FIG. 1. The ground-state energy $E_{g}$ as a function of the coupling $g$ for the CCM SUB- $N$ approximations with $N=10,30,50$, for the case $\omega=\omega_{0}=1$. Also shown are the results of an "exact" diagonalization in a basis of 100 positive-parity states.

coupling by using the solution at the previous coupling as input to the iterative routine for solving the equations for the coefficients. For simplicity, we will only quote results for the case of a scaled Hamiltonian at resonance $\left(\omega=\omega_{0}=1\right)$.

We find that the ground-state energy shows evidence for spontaneous breaking of the parity symmetry. For each $N$, the solution with positive parity (signalled by $S_{2 k+1}^{(1)}=S_{2 k+1}^{(2)}=0$ for $\left.k=0,1,2 \ldots\right)$ terminates at a finite value of $g$, which we indicate by $g_{c}^{(N)}$; the solution above this coupling has mixed symmetry. However, it is important to realize that it would be dangerous to trust our method to produce realistic results in the broken-parity regime, since our model state is of positive parity. To describe the system correctly in the broken-parity regime, a mixed-parity model state would be required. This situation is very similar to that seen in the CCM analysis of the Lipkin-Meshkov-Glick model [8].

There is a difference in the nature of the termination of the positive-parity solutions depending on whether $N / 2$ is odd or even. It is easiest to examine this difference by referring to the quantity $\chi=-d^{2} E_{g} / d g^{2}$, which can be termed a coupling susceptibility. For $N / 2$ even, the approximant $\chi^{(N)}$ as a function of $g$ starts positive at $g=0$ and displays a (local) minimum of finite depth followed by a (local) maximum. It then seems to diverge to $-\infty$, although it becomes progressively more difficult to track the solution for either large $N$ or large $g$. The value of $\chi^{(N)}$ at the local minimum appears to diverge to $-\infty$ in the limit $N \rightarrow \infty$. For $N / 2$ odd, the situation is somewhat simpler. We find that $\chi^{(N)}$ displays a divergence to $+\infty$ for finite values of $N$. Due to this rather more straightforward behavior, we shall restrict further discussion to this case.

In Fig. 1 we show the ground-state energy $E_{g}$ as a function of the coupling $g$ for resonance $\left(\omega=\omega_{0}=1\right)$ for several SUB- $N$ approximations, together with the "exact" results from a diagonalization of (1) in a basis of 100 positive-parity states. For $g<g_{c}^{(N)}$ the agreement between the results of the CCM calculations and the diagonalization is very good. The termination points of the CCM solutions with positive parity are clearly visible. 


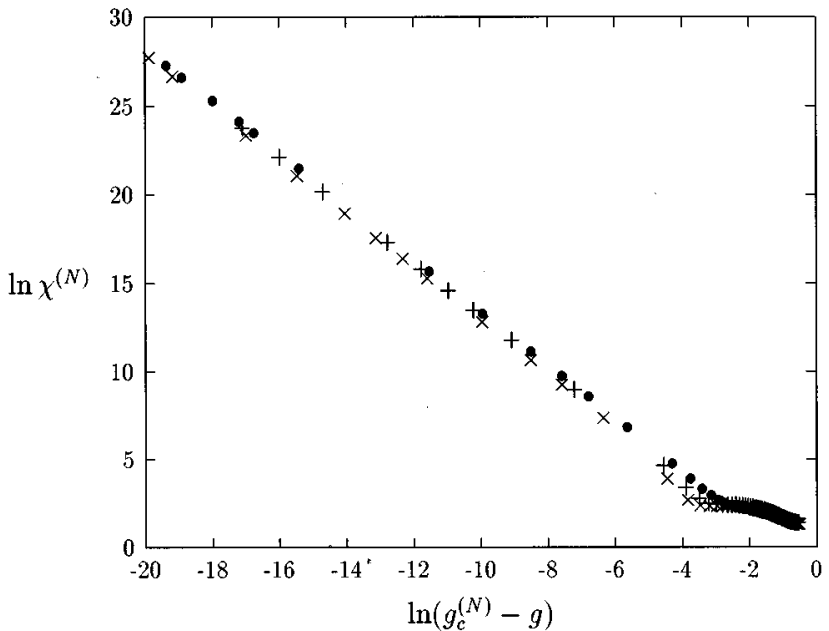

FIG. 2. $\ln \chi^{(N)}$ as a function of $\ln \left[g_{c}^{(N)}-g\right]$ for the CCM SUB- $N$ approximations with $N=10$ (dots), 30 (plus signs), and 50 (crosses), for the case $\omega=\omega_{0}=1$.

It is of interest to examine the nature of the divergence of $\chi^{(N)}$ so as to obtain values for the critical exponent of $E_{g}$. To this end, we have fitted $\ln \chi^{(N)}$ to a function of the form $m^{(N)} \ln \left[g_{c}^{(N)}-g\right]+\ln c^{(N)}$ for several values of $N$ (see Fig. 2). For $g \rightarrow\left(g_{c}^{(N)}\right)^{-}$, we consistently obtain $m^{(N)} \approx-3 / 2$, the uncertainty arising from the fact that $g_{c}^{(N)}$ is not known precisely. This strongly suggests that for $g$ just below the critical coupling the ground-state energy at the SUB- $N$ level has an expansion of the form

$$
E_{g}^{(N)}=f_{0}^{(N)}+f_{1}^{(N)}\left(g_{c}^{(N)}-g\right)^{1 / 2}+f_{2}^{(N)}\left(g_{c}^{(N)}-g\right)+\cdots,
$$

where $\left\{f_{i}^{(N)}\right\}$ are constants that depend on the level of truncation.

The question of the behavior of $\chi$ and $E_{g}$ in the limit $N \rightarrow \infty$ is more subtle, due to the fact that, although $m^{(N)}$ appears to be independent of $N, c^{(N)}$ does show such a dependence. To investigate this further, we have made use of a modification of the coherent anomaly method (CAM) of Suzuki [9] to perform the extrapolation $N \rightarrow \infty$. The essential feature of this method is the parametrization of the intercept $c^{(N)}$ as $c\left(g_{c}-g_{c}^{(N)}\right)^{\nu}$, where $g_{c} \equiv g_{c}^{(\infty)}$ and $c$ is independent of $N$. This implies that in the (exact) $N \rightarrow \infty$ limit, we have $\chi \sim\left(g_{c}-g\right)^{-3 / 2+\nu}$, so that in the limit $g \rightarrow\left(g_{c}\right)^{-}$the groundstate energy behaves as

$$
E_{g}=f_{0}+f_{1}\left(g_{c}-g\right)+f_{2}\left(g_{c}-g\right)^{1 / 2+\nu}+\cdots,
$$

where $\left\{f_{i}\right\}$ are constants. The "anomalous" exponent $\nu$ is then found by plotting $\ln \left[\chi^{(N)}\left(g_{c}^{(N)}-g\right)^{3 / 2}\right]$ against $\ln \left[g_{c}-g_{c}^{(N)}\right]$.

To carry out the CAM analysis, we therefore require an estimate of $g_{c}$. In Fig. 3, we plot $g_{c}^{(N)}$ vs $N$ for $N \leqslant 98$ ( $N$ even, $N / 2$ odd). There appears to be convergence in the limit $N \rightarrow \infty$. To obtain an estimate of the exact critical coupling $g_{c}^{(\infty)}$, we have performed a least-squares fit of $g_{c}^{(N)}$ to the form $a-b N^{\gamma}$ for $N$ larger than some minimum value, say $N_{\min }$. Numerical studies indicate that a choice of $\gamma=-2 / 3$ yields consistency with the $-3 / 2$ critical exponent found for $\chi^{(N)}$. We have thus fixed $\gamma$ to this value. The

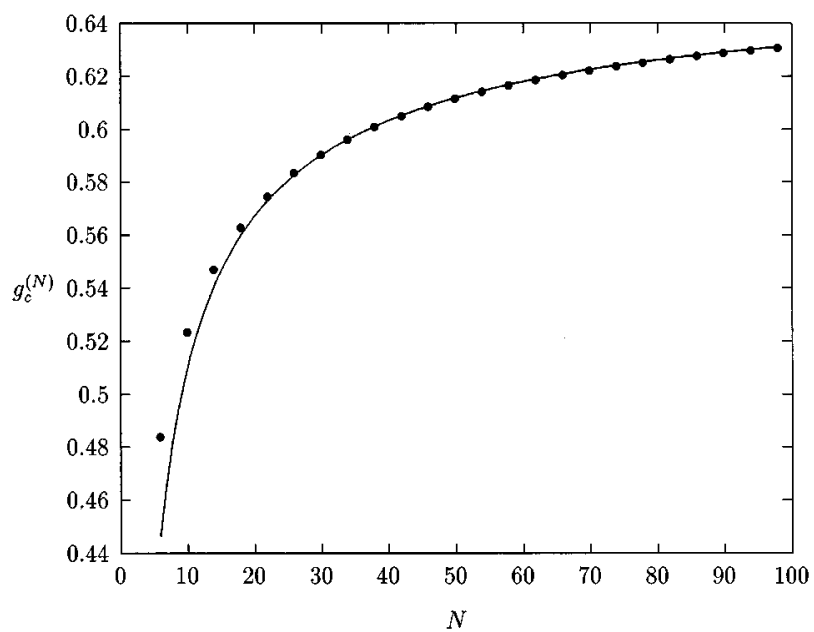

FIG. 3. The critical coupling $g_{c}^{(N)}$ as a function of the level of CCM approximation $N$, for the case $\omega=\omega_{0}=1$. The solid line is the function $0.66494-0.7218 N^{-2 / 3}$ obtained from a least-squares fit to $g_{c}^{(N)}$ for $62 \leqslant N \leqslant 98$.

resultant fits are very insensitive to the precise choice of $N_{\min }$ so long as $N_{\min }$ is large enough, say $N_{\min }>50$. A representative fit is that obtained for $N_{\min }=62$, where we obtain $a=0.66494 \pm 0.00001$ and $b=0.7218 \pm 0.0003$. This leads us to suggest that in the (exact) SUB- $\infty$ limit there is a parity-breaking transition at a coupling of $g_{c} \sim 0.665$.

Using this value for $g_{c}$ in the CAM analysis, we obtain $\nu=0.53 \pm 0.01$, with a ground-state energy given by Eq. (8) near $g_{c}$. The closeness of our calculated value for the exponent $\nu$ to $1 / 2$ leads us to speculate that the ground-state energy may actually have an essential (not a pure power-law) singularity at $g=g_{c}$, with $E_{g}$ and $d E_{g} / d g$ continuous (as required by the Hellmann-Feynman theorem). Similar behavior is certainly known to exist in some comparable exactly integrable models. A prime example is the quantum phase transition at the isotropic Heisenberg point for a onedimensional (1D) chain of spin-1/2 atoms interacting via the nearest-neighbor anisotropic Heisenberg (or $X X Z$ ) Hamiltonian, which is exactly soluble by Bethe ansatz techniques [10]. This phase transition has an essential singularity at which $E_{g}$ and $d^{n} E_{g} / d g^{n}$ are continuous for all finite values of the positive integer $n$. Such cases with very subtle phase transitions are often difficult to treat by approximate methods. However, we note that the CCM has been applied to this model in both 1D and 2D, and equally strong evidence for similar critical behavior has also been observed in various truncation schemes [11].

An obvious candidate for the order parameter associated with the phase transition is $\left\langle\sigma^{x}\right\rangle$. In the NCCM its value is given by

$$
\begin{aligned}
\left\langle\sigma^{x}\right\rangle= & 2 \widetilde{S}_{1}^{(2)}+2\left[S_{1}^{(2)}+\sum_{n=1}^{\infty} \widetilde{S}_{n}^{(1)} S_{n+1}^{(2)}\right] \\
& -8 \sum_{n=1}^{\infty} \sum_{m=1}^{\infty}(n+m-2) ! S_{n}^{(2)} S_{m}^{(2)} \widetilde{S}_{n+m-1}^{(2)} .
\end{aligned}
$$

Below the transition, $\left\langle\sigma^{x}\right\rangle=0$ for any positive-parity state due to the fact that the odd-indexed coefficients are all zero. 
In the limit $\omega_{0} \rightarrow 0$ (or equivalently $g \rightarrow \infty$ ), it is simple to see that $\left\langle\sigma^{x}\right\rangle \rightarrow 2 S_{1}^{(2)} \rightarrow \pm 1$, as can be predicted from the fact that $\left[H, \sigma^{x}\right] \rightarrow 0$ in this limit. Since our choice of model state precludes an accurate description of the broken-parity phase, it is not sensible to calculate $\left\langle\sigma^{x}\right\rangle$ for this phase in our present work.

Most work to date on the Jaynes-Cummings model beyond the RWA treats the counterrotating terms included in our Rabi Hamiltonian (1) either as sources of slight frequency shifts or phase-dependent corrections or as sources of chaotic motion [3]. Carmichael and co-workers [12] examined the Rabi Hamiltonian with an additional coupling of the boson mode to an external field. After making the RWA, they found a transition from a discrete to a continuous spectrum as a function of the strength of the external field. One should note, however, that for these authors the strongcoupling regime means that the dipole coupling constant $g$ is comparable to or larger than the radiative linewidths, whereas in our case strong coupling means that $g$ becomes comparable to $\omega$ and $\omega_{0}$. At typical optical or micromaser frequencies the two regimes are six or so orders of magnitude apart, and the phase transition observed by us is not obviously related to the transition observed by Carmichael and co-workers.
By contrast to the perturbative or semiclassical treatments of previous work on the Rabi Hamiltonian, we have applied the CCM to the calculation of its ground-state energy. We have seen that for the weak-coupling range of parameters typical of optical applications the method converges extremely rapidly to yield very accurate results. However, since quantum optics experiments are nowadays being performed with ever-increasing field intensities, we concur with other authors (see, e.g., [13]) that it is of great interest to explore the full Rabi Hamiltonian with a nonperturbative approach with a proven track record, such as the CCM. One outcome has been that for stronger couplings than have hitherto been explored experimentally, our results provide strong evidence for a second-order quantum phase transition. In particular, for the special case of resonance, we predict the transition to take place at a coupling (scaled by the frequency of the electromagnetic field) of approximately 0.665. Calculations away from resonance suggest that the qualitative behavior for this situation is similar.

We acknowledge useful discussions with A. Vourdas, H. Kümmel, and H. J. Carmichael. R.F.B. acknowledges the support of a research grant from the EPSRC, Great Britain. R.M.Q. and D.vdW. acknowledge the financial support of the FRD, South Africa.
[1] E. T. Jaynes and F. W. Cummings, Proc. IEEE 51, 89 (1963).

[2] P. W. Milonni and S. Singh, Adv. At. Mol. Opt. Phys. 28, 75 (1991); B. W. Shore and P. L. Knight, J. Mod. Opt. 40, 1195 (1993).

[3] P. W. Milonni, J. R. Ackerhalt, and H. W. Galbraith, Phys. Rev. Lett. 50, 966 (1983); M. Kuś, ibid. 54, 1343 (1985).

[4] H. G. Reik, P. Lais, M. E. Stützle, and M. Doucha, J. Phys. A 20, 6327 (1987).

[5] W. H. Wong and C. F. Lo, Phys. Rev. B 50, 17615 (1994).

[6] R. F. Bishop, Theor. Chim. Acta. 80, 95 (1991).

[7] J. Arponen, Ann. Phys. (N.Y.) 151, 311 (1983).

[8] J. Arponen and J. Rantakivi, Nucl. Phys. A407, 141 (1983); N.
I. Robinson, R. F. Bishop, and J. Arponen, Phys. Rev. A 40, 4256 (1989).

[9] M. Suzuki, J. Phys. Soc. Jpn. 55, 4205 (1986).

[10] H. A. Bethe, Z. Phys. 71, 205 (1931); L. Hulthén, Ark. Mat. Astron. Fys. A 26 (11) (1938).

[11] R. F. Bishop, J. B. Parkinson, and Y. Xian, Phys. Rev. B 44, 9425 (1991); R. F. Bishop, R. G. Hale, and Y. Xian, Phys. Rev. Lett. 73, 3157 (1994).

[12] P. Alsing and H. J. Carmichael, Quantum Opt. 3, 13 (1991); P. Alsing, D. S. Guo, and H. J. Carmichael, Phys. Rev. A 45, 5135 (1992).

[13] M. D. Crisp, Phys. Rev. A 43, 2430 (1991). 